\title{
Non-real eigenvalues of nonlocal indefinite Sturm-Liouville problems
}

\author{
Fu Sun ${ }^{1}$, Kun Li²* Jiangang Qi ${ }^{1}$ and Baochao Liao ${ }^{1}$
}

$\bar{~}{ }^{\text {Correspondence: qslikun@163.com }}$ ${ }^{2}$ School of Mathematical Sciences, Qufu Normal University, Qufu, P.R. China

Full list of author information is available at the end of the article

\begin{abstract}
The present paper deals with non-real eigenvalues of regular nonlocal indefinite Sturm-Liouville problems. The existence of non-real eigenvalues of indefinite Sturm-Liouville differential equation with nonlocal potential $K(x, t)$ associated with self-adjoint boundary conditions is studied. Furthermore, a priori upper bounds of non-real eigenvalues for a class of indefinite differential equation involving nonlocal point interference potential function is obtained.
\end{abstract}

MSC: 34B24; 34L15; 47B50

Keywords: Indefinite Sturm-Liouville problem; Nonlocal potential; Non-real eigenvalue; A priori bounds

\section{Introduction}

Consider the regular nonlocal indefinite Sturm-Liouville differential equation

$$
-y^{\prime \prime}(x)+q(x) y(x)+\int_{-1}^{1} K(x, t) y(t) \mathrm{d} t=\lambda w(x) y(x) \quad \text { in } L_{|w|}^{2}[-1,1]
$$

associated to suitable boundary conditions, where $\lambda$ is the spectral parameter, $q \in L^{1}[-1,1]$ is called the local potential, $K(x, t)=\overline{K(t, x)}$ is called the nonlocal potential, the weight function $w \in L^{1}[-1,1]$ changes its sign on $[-1,1]$ in the sense that mes $\{x \in[-1,1]: w(x)>$ $0\}$ and $\operatorname{mes}\{x \in[-1,1]: w(x)<0\}$ have a positive Lebesgue measure and $L_{|w|}^{2}:=L_{|w|}^{2}[-1,1]$ is the weighted Hilbert space of all Lebesgue measurable, complex-valued functions $f$ on $[-1,1]$ satisfying $\int_{-1}^{1}|w||f|^{2}<\infty$ with the inner product $(f, g)_{|w|}=\int_{-1}^{1}|w| f \bar{g}$ and the norm $\|f\|_{|w|}^{2}=\int_{-1}^{1}|w||f|^{2}$. Such a problem (1.1) is called indefinite.

If $w(x)>0$ a.e. $x \in[-1,1]$, models similar to the nonlocal differential equation (1.1) appear in quantum mechanics, diffusion processes, point interactions, voltage-driven electrical systems and have been studied in $[2,15,27]$. In the case where $q \equiv 0, w \equiv 1$ and $K(x, t)=v(x) u(t), v, u \in C([-1,1], \mathbb{R})$ in (1.1), the authors in [12] investigate the reality of eigenvalues with Dirichlet boundary conditions. For the case

$$
K(x, t)=v(x) \delta(t-c)+\overline{v(t)} \delta(x-c), \quad c \in(-1,1],
$$

where $v \in L^{2}([-1,1], \mathbb{C}), \delta$ is Dirac's distribution, the inverse spectral problems for various nonlocal operators were studied in $[1,19,20]$ and the references cited therein.

(c) The Author(s) 2019. This article is distributed under the terms of the Creative Commons Attribution 4.0 International License (http://creativecommons.org/licenses/by/4.0/), which permits unrestricted use, distribution, and reproduction in any medium, provided you give appropriate credit to the original author(s) and the source, provide a link to the Creative Commons license, and indicate if changes were made. 
The nonlocal indefinite Sturm-Liouville problems have attracted a lot of attention in recent years since the diversity and complexity of biomathematics models, transport models, population dynamic systems and microwave propagation problems. Such nonlocal problems also played an important role in reaction-diffusion problems and quantummechanical theory. For the case $K(x, t)$ in (1.2) with suitable boundary conditions, the spectral problems which including the change rule of (non-)left-definiteness for the local case under the nonlocal perturbations, the equivalence of non-left-definite and the finiteness of non-real eigenvalues of nonlocal indefinite Sturm-Liouville equation (1.1) have been studied in [26]. The (local) indefinite Sturm-Liouville problem, i.e., $K(x, t)=0$ in (1.1) with self-adjoint boundary conditions, has discrete, real eigenvalues unbounded from both below and above, and may also admit non-real eigenvalues (see [3, 16, 18, 29]). The indefinite nature was noticed by Haupt [13], Richardson [24] at the beginning of the last century and has attracted a lot of attention in recent years. Determining a priori bounds and determining the exact number of non-real eigenvalues are an interesting and difficult problems in Sturm-Liouville theory. Recently, these open problems have been solved by Qi et al., [4, 14, 21, 28] for the regular (local) indefinite problem and by Behrndt et al., [5-9, 25] for the singular case, respectively.

In the present paper, we firstly obtain the existence of the nonlocal indefinite differential equation (1.1) with self-adjoint boundary conditions by means of the operator theory in Krein spaces and the symmetry conditions. In the case of the equation with a nonlocal point interference potential function (Dirac distribution), we derive an equation without Dirac's distribution but with a nonlocal boundary condition. Then we study the estimates on the upper bounds of non-real eigenvalues for this nonlocal indefinite Sturm-Liouville problem.

The arrangement of this paper is as follows: Sect. 2 is devoted to the existence of nonreal eigenvalues for the nonlocal indefinite Sturm-Liouville equation (1.1) associated with self-adjoint separated boundary conditions (see Theorem 2.2). In Sect. 3, we obtain the nonlocal indefinite Sturm-Liouville problem through the Dirac distribution in (1.2), then the upper bounds of non-real eigenvalues in terms of $q, v, w$ are shown (see Theorems 4.1 and 4.2) in Sect. 4.

\section{Existence of non-real eigenvalues}

In this section we prove the existence of non-real eigenvalues for the nonlocal indefinite eigenvalue problem

$$
\left\{\begin{array}{l}
\tau_{k} y:=-y^{\prime \prime}(x)+q(x) y(x)+\int_{-1}^{1} K(x, t) y(t) \mathrm{d} t=\lambda w(x) y(x), \\
\mathcal{B}_{k} y=0: \quad y(-1)=0, \quad y^{\prime}(1)=0,
\end{array}\right.
$$

where $w$ changes sign on $[-1,1]$ satisfying

$$
w(x) \neq 0 \quad \text { a.e. } x \in[-1,1], q, w \in L^{1}([-1,1], \mathbb{R}) \text { and } K \in L^{1}\left([-1,1]^{2}, \mathbb{R}\right) .
$$

To this end, we need the following lemma.

Lemma 2.1 If $w \in L^{1}([-1,1], \mathbb{R})$ and $|w(x)|>0$ a.e. on $[-1,1]$, then the eigenvalue problems $\tau_{k} y=\lambda|w| y, \mathcal{B}_{k} y=0$ and $\tau_{k} y=\lambda y, \mathcal{B}_{k} y=0$ have the same number of negative eigenvalues. 
Proof Let $\tilde{S}_{k}$ and $\tilde{S}$ be the operator associated to

$$
\tau_{k} y=\lambda|w| y, \quad \mathcal{B}_{k} y=0 \quad \text { and } \quad \tau_{k} y=\lambda y, \quad \mathcal{B}_{k} y=0
$$

where $D\left(\tilde{S}_{k}\right)=\left\{y \in L_{|w|}^{2}: y, y^{\prime} \in A C_{\text {loc }}, \tau_{k} y /|w| \in L_{|w|}^{2}, \mathcal{B}_{k} y=0\right\}$ and $D(\tilde{S})=\left\{y \in L^{2}: y, y^{\prime} \in\right.$ $\left.A C_{\text {loc }}, \tau_{k} y \in L^{2}, \mathcal{B}_{k} y=0\right\}$, respectively. Note that $D\left(\tilde{S}_{k}\right)=D(\tilde{S})$, one sees that for every $y \in D\left(\tilde{S}_{k}\right),\left(\tilde{S}_{k} y, y\right)_{|w|}=(\tilde{S} y, y)_{L^{2}}$. It follows from the Min-Max principle for self-adjoint operators [23, Theorem XIII.1, p. 76] that

$$
\mu_{n}=\sup _{g_{1}, \ldots, g_{n-1} f \in\left[g_{1}, \ldots, g_{n-1}\right]^{\perp}} \inf \left\{\frac{(S f, f)_{|w|}}{(f, f)_{|w|}}: f \neq 0, f \in D(S)\right\}
$$

and that the negative eigenvalues are dependent only on the quadratic form of operator $(S f, f)_{|w|}$. Hence $\tilde{S}_{k}$ and $\tilde{S}$ have the same number of negative eigenvalues, which completes the proof of Lemma 2.1 .

In what follows, we impose the symmetry conditions on $q, K$ and $w$, namely,

$$
q(x)=q(-x), \quad K(x, t)=K(-x, t), \quad w(-x)=-w(x)
$$

to prove the existence of non-real eigenvalues of (2.1). It follows from the hypothesis on $q, K, w$ in (2.2) and the symmetry conditions (2.3) that if $\lambda \in \mathbb{C}$ is an eigenvalue of the problem (2.1) and $\phi$ is the corresponding eigenfunction, then $-\bar{\lambda}$ is also an eigenvalue of (2.1) with eigenfunction $\overline{\phi(-\cdot)}$. Let $\tilde{K}=\left(L_{|w|}^{2},[\cdot, \cdot]_{w}\right)$ be the Krein space equipped with the indefinite inner product $[f, g]_{w}=\int_{-1}^{1} w f \bar{g}, f, g \in L_{|w|}^{2}$ and $T$ the self-adjoint operator in $\tilde{K}$ (cf. $[10,11])$ defined as

$$
T y=\frac{1}{w} \tau_{k} y, \quad y \in D(T)=\left\{y \in L_{|w|}^{2}: y, y^{\prime} \in A C_{\mathrm{loc}}[-1,1], \tau_{k} y /|w| \in L_{|w|}^{2}, \mathcal{B}_{k} y=0\right\} .
$$

We say that the self-adjoint operator $T$ has $k$ negative squares, $k \in \mathbb{N}_{0}$, if there exists a $k$-dimensional subspace $X$ of $\tilde{K}$ in $D(T)$ such that $[T f, f]<0, f \in X, f \neq 0$, but no $(k+1)$ dimensional subspace with this property.

Applying the above results and the spectral theory of operators in $\tilde{K}$ spaces, we will prove the existence of non-real eigenvalues of (2.1) by the method given in [21].

Theorem 2.2 Let (2.2) and (2.3) hold. If the eigenvalue problem

$$
-y^{\prime \prime}(x)+q(x) y(x)+\int_{-1}^{1} K(x, t) y(t) \mathrm{d} t=\lambda y(x), \quad \mathcal{B}_{k} y=0,
$$

has one negative eigenvalue and the remaining eigenvalues are all positive, then the nonlocal indefinite Sturm-Liouville problem (2.1) has two non-real eigenvalues.

Proof Let $T_{k}$ and $S_{k}$ be the operators of the nonlocal indefinite Sturm-Liouville problem (2.1) and nonlocal right-definite Sturm-Liouville problem $\tau_{k} y=\lambda|w| y, \mathcal{B}_{k} y=0$, respectively. Then $T_{k}$ and $S_{k}$ are self-adjoint under the indefinite inner product $[\cdot, \cdot]_{w}$ and the definite inner product $(\cdot, \cdot)_{|w|}$, respectively. This together with Lemma 2.1 and the 
assumption in Theorem 2.2 shows that $S_{k}$ has one negative eigenvalue and the rest are all positive, therefore, $T_{k}$ has exactly one negative square because of $\left[T_{k} f, f\right]_{w}=\left(S_{k} f, f\right)_{|w|}$ and 0 is a resolvent point of $T_{k}$. It follows from [11, Proposition 1.5] that there exists exactly one eigenvalue $\lambda$ of (2.1) in $\mathbb{R}$ or the upper half-plane $\mathbb{C}$ and if this eigenvalue $\lambda \in \mathbb{R}$ then there exists an eigenfunction $\phi$ with $\lambda[\phi, \phi]_{w} \leq 0$. Let $\lambda \in \mathbb{R}$ be such an eigenvalue with eigenfunction $\phi$, then $-\lambda=-\bar{\lambda}$ is also an eigenvalue with the eigenfunction $\overline{\phi(-\cdot)}$ and $-\lambda[\overline{\phi(-\cdot)}, \overline{\phi(-\cdot)}]_{w}=\lambda[\phi, \phi]_{w} \leq 0$ through the symmetry in (2.3). Hence we get two eigenvalues, which is a contradiction. If $\lambda \in \mathbb{C}^{+}$, then $-\bar{\lambda} \in \mathbb{C}^{+}$, which implies that $\lambda=-\bar{\lambda}$, i.e., $\lambda$ is purely imaginary. The proof is finished.

\section{Nonlocal Sturm-Liouville problems with distribution coefficients}

Let the kernel $K(x, t)$ in (1.2) be given in the form

$$
K(x, t)=v(x) \delta(t-c)+\overline{v(t)} \delta(x-c), \quad c \in(-1,1]
$$

where $v \in L^{1}([-1,1], \mathbb{R})$ and $\delta$ is Dirac's distribution. For every continuous function $f$ on $[-1,1]$, the Dirac delta distribution at point $c$ is defined by

$$
\int_{-1}^{1} \delta(x-c) f(x) \mathrm{d} x= \begin{cases}f(c), & c \in[-1,1] \\ 0, & c \notin[-1,1]\end{cases}
$$

In this case, a solution of (1.1) is understood in the sense that a function $y \in A C[-1,1]$, $y^{\prime} \in A C([-1, c) \cup(c, 1])$ such that $y^{\prime}(c \pm 0)$ exist and the equation holds almost everywhere. For $c=1$, we use $y^{\prime}(1)$ instead of $y^{\prime}(1+0)$. It follows from (3.1), (3.2) and the continuity of the solution $y$ that (1.1) takes the form

$$
-y^{\prime \prime}(x)+q(x) y(x)+v(x) y(c)+\delta(x-c) \int_{-1}^{1} v(t) y(t) \mathrm{d} t=\lambda w(x) y(x)
$$

for a.e. $x \in[-1,1]$. For $x \in[-1,1]$ and $x \neq c$, the equation has the form

$$
-y^{\prime \prime}(x)+q(x) y(x)+v(x) y(c)=\lambda w(x) y(x) \quad \text { a.e. } x \in[-1,1] .
$$

Integrating both sides of (3.3) on the interval $[c-\varepsilon, c+\varepsilon]$ for arbitrary $\varepsilon>0$, then

$$
y^{\prime}(c-\varepsilon)-y^{\prime}(c+\varepsilon)+\int_{-1}^{1} v(t) y(t) \mathrm{d} t=\int_{c-\varepsilon}^{c+\varepsilon}((\lambda w(x)-q(x)) y(x)-v(x) y(c)) \mathrm{d} x .
$$

Let $\varepsilon \rightarrow 0$, one sees that $y^{\prime}(c-0)-y^{\prime}(c+0)+\int_{-1}^{1} v(x) y(x) \mathrm{d} x=0$. Then $y$ satisfies

$$
\left\{\begin{array}{l}
-y^{\prime \prime}(x)+q(x) y(x)+v(x) y(c)=\lambda w(x) y(x) \quad \text { a.e. } x \in[-1,1], x \neq c, \\
y^{\prime}(c-0)-y^{\prime}(c+0)+\int_{-1}^{1} v(x) y(x) \mathrm{d} x=0 .
\end{array}\right.
$$

If the boundary condition is given in the form $y(-1)=0, y^{\prime}(1)=0$ for $(1.1)$ and let $c=1$, then from (3.4) we see that the nonlocal indefinite eigenvalue problem takes the form

$$
\left\{\begin{array}{l}
-y^{\prime \prime}(x)+q(x) y(x)+v(x) y(1)=\lambda w(x) y(x) \\
y(-1)=0, \quad y^{\prime}(1-0)+\int_{-1}^{1} v(x) y(x) \mathrm{d} x=0
\end{array}\right.
$$


The authors in $[1,19]$ investigate the eigenvalue problem with $q \equiv 0, w \equiv 1$. For simplicity, we will write $y^{\prime}(1)$ instead of $y^{\prime}(1-0)$ in the following discussion.

\section{A priori bounds of non-real eigenvalues}

Consider the nonlocal indefinite Sturm-Liouville problem

$$
\left\{\begin{array}{l}
\tau y:=-y^{\prime \prime}(x)+q(x) y(x)+v(x) y(1)=\lambda w(x) y(x), \\
\mathcal{B} y=0: \quad y(-1)=0, \quad y^{\prime}(1)+\int_{-1}^{1} v(x) y(x) \mathrm{d} x=0,
\end{array}\right.
$$

where $q, v, w$ are real-valued functions satisfying the standard conditions

$$
w(x) \neq 0 \quad \text { a.e. } x \in[-1,1], q, v, w \in L^{1}[-1,1] .
$$

The operator $S$ associated to the nonlocal right-definite problem

$$
-y^{\prime \prime}(x)+q(x) y(x)+v(x) y(1)=\lambda|w(x)| y(x), \quad \mathcal{B} y=0,
$$

is defined as $S y=\frac{1}{|w|} \tau y$ for $y \in D(S)$, where

$$
D(S)=\left\{y \in L_{|w|}^{2}: y, y^{\prime} \in A C_{\mathrm{loc}}[-1,1], \tau y /|w| \in L_{|w|}^{2}, \mathcal{B} y=0\right\} .
$$

It follows from [22] that $S$ is a self-adjoint operator in the Hilbert space $\left(L_{|w|}^{2},(\cdot, \cdot)_{|w|}\right)$ and its spectrum consists of real eigenvalues, which are bounded from below.

To simplify our statements, let $\|\cdot\|_{p}$ be the $L^{p}[-1,1]$-norm, $1 \leq p<\infty,\|\cdot\|_{\infty}$ be the $L^{\infty}[-1,1]$-norm and

$$
N_{q, v}=1+8\left(\left\|q_{-}\right\|_{1}+8\|v\|_{1}^{2}\right), \quad q_{-}=\max \{-q, 0\},\|q\|_{1}=\int_{-1}^{1}|q|,\|v\|_{1}=\int_{-1}^{1}|v| .
$$

If $x w(x)>0$ a.e. on $[-1,1]$, we can choose $\varepsilon>0$ such that

$$
\Omega(\varepsilon)=\{x \in[-1,1]: x w(x)<\varepsilon\}, \quad m(\varepsilon)=\operatorname{mes} \Omega \leq \frac{1}{4 N_{q, v}} .
$$

Since $w^{2}(x)>0$ a.e. on $[-1,1]$, we can choose $\eta>0$ such that

$$
\widetilde{\Omega}(\eta)=\left\{x \in[-1,1]: w^{2}(x)<\eta\right\}, \quad m(\eta)=\operatorname{mes} \widetilde{\Omega} \leq \frac{1}{4 N_{q, v}} .
$$

A point at $x$ which the weight function $w(x)$ changes its sign will be called a turning point [16]. If $w(x)$ has only one turning point, we can obtain the following a priori bounds on the non-real eigenvalues.

Theorem 4.1 Assume that $x w(x)>0$ a.e. on $[-1,1]$ and (4.2) holds, then, for any possible non-real eigenvalue $\lambda$ of problem (4.1), we have

$$
|\lambda| \leq \frac{2}{\varepsilon}\left(\sqrt{N_{q, v}}+4 N_{q, v}\left(\left\|q_{-}\right\|_{1}+2\|v\|_{1}\right)\right), \quad|\operatorname{Im} \lambda| \leq \frac{2}{\varepsilon}\left(\sqrt{N_{q, v}}+8 N_{q, v}\|v\|_{1}\right),
$$

where $\varepsilon>0$ and $q_{-}, N_{q, v}$ are defined in (4.5) and (4.4), respectively. 
If $w(x)$ is allowed to have more than one turning point, we will obtain the following results.

Theorem 4.2 Assume that $w \in A C[-1,1], w^{\prime} \in L^{2}[-1,1]$ and (4.2) holds, then, for any nonreal eigenvalue $\lambda$ of problem (4.1), we have

$$
\begin{aligned}
& |\lambda| \leq \frac{2}{\eta}\left(\|w\|_{\infty} N_{q, v}\left(1+2\left\|q_{-}\right\|_{1}+4\|v\|_{1}\right)+\Lambda \sqrt{N_{q, v}}\right), \\
& |\operatorname{Im} \lambda| \leq \frac{2}{\eta}\left(4\|w\|_{\infty}\|v\|_{1} N_{q, v}+\Lambda \sqrt{N_{q, v}}\right),
\end{aligned}
$$

where $\Lambda=\left(\int_{-1}^{1}\left|w^{\prime}\right|^{2}\right)^{\frac{1}{2}}, \eta>0$ and $q_{-}, N_{q, v}$ are defined in (4.6) and (4.4), respectively.

In order to prove Theorems 4.1 and 4.2, we first introduce some concepts and prepare some lemmas (cf. [17]). Let $f$ be a real-valued function defined on the closed, bounded interval $[a, b]$ and $\Delta: a=x_{0}<x_{1}<\cdots<x_{n}-1<x_{n}=b$ be a partition of $[a, b]$. We define the variation of $f$ with respect to $\triangle$ by

$$
\operatorname{Var}_{\triangle}=\sum_{i=1}^{n}\left|f\left(x_{i}\right)-f\left(x_{i-1}\right)\right|
$$

and the total variation of $f$ on $[a, b]$ by

$$
\bigvee_{a}^{b}(f)=\sup \left\{\operatorname{Var}_{\Delta}: \triangle \text { is an any partition of }[a, b]\right\}
$$

A real-valued function $f$ is said to be of bounded variation on the closed, bounded interval $[a, b]$ if $\bigvee_{a}^{b}(f)<\infty$.

Lemma 4.3 (cf. [14, Lemma 2] and [17, Lemma 5.2.2, p. 246]) Let $g$ be of bounded variation over all of $[a, b]$, that is, $g$ satisfies the inequality $\int_{a}^{x}|\mathrm{~d} g(x)|<\infty$. Then for all $x \in(a, b]$ and for every $\delta>0$ there exists a $\rho=\rho(\delta, x)>0$ such that

$$
\int_{a}^{x}|f(t)|^{2}|\mathrm{~d} g(t)| \leq \rho(\delta, x) \int_{a}^{x}|f(t)|^{2} \mathrm{~d} t+\delta \int_{a}^{x}\left|f^{\prime}(t)\right|^{2} \mathrm{~d} t
$$

where

$$
\rho(\delta, x)=\frac{1}{x-a}+\frac{c}{\delta}, \quad c=\int_{a}^{b}|\mathrm{~d} g(x)| .
$$

Lemma 4.4 Let $q, v \in L^{1}[-1,1]$ and $\phi \in D(T)$. Then

$$
\int_{-1}^{1}\left|q_{-}+\sqrt{2} \varepsilon\|v\|_{1}\right| v|||\phi|^{2} \leq\left(\frac{1}{2}+\frac{\left\|q_{-}\right\|_{1}+\sqrt{2} \varepsilon\|v\|_{1}^{2}}{\delta}\right) \int_{-1}^{1}|\phi|^{2}+\delta \int_{-1}^{1}\left|\phi^{\prime}\right|^{2} .
$$


Proof Replacing $f(t)$ and $g(t)$ by $\phi(t)$ and $\int_{-1}^{t}\left(q_{-}(x)+\sqrt{2} \varepsilon\|v\|_{1}|v(x)|\right) \mathrm{d} x$ in Lemma 4.3, respectively, then

$$
\begin{aligned}
\int_{-1}^{x}|\mathrm{~d} g(t)| & =\int_{-1}^{x}\left|\mathrm{~d}\left(\int_{-1}^{t}\left(q_{-}(x)+\sqrt{2} \varepsilon\|v\|_{1}|v(x)|\right) \mathrm{d} x\right)\right| \\
& =\int_{-1}^{x}\left|q_{-}(t)+\sqrt{2} \varepsilon\|v\|_{1}\right| v(t)\|\mathrm{d} t \leq\| q_{-}\left\|_{1}+\sqrt{2} \varepsilon\right\| v \|_{1}^{2}<\infty
\end{aligned}
$$

Using this result in (4.9), one sees that (4.10) holds immediately.

The following lemma presents estimates of $\left\|\phi^{\prime}\right\|_{2}$ and $\|\phi\|_{\infty}$, where $\phi$ is an eigenfunction of (4.1) corresponding to a non-real eigenvalue $\lambda$. That is, $\mathcal{B} \phi=0$ and

$$
-\phi^{\prime \prime}+q \phi+\nu \phi(1)=\lambda w \phi .
$$

Since the problem (4.1) is a linear system and $\phi$ is continuous, we can choose $\phi$ to satisfy $\int_{-1}^{1}|\phi(x)|^{2} \mathrm{~d} x=1$ in the following discussion.

Lemma 4.5 Let $\lambda$, $\phi$ be defined as above and $N_{q, v}$ in (4.4). Then

$$
\left\|\phi^{\prime}\right\|_{2}^{2} \leq N_{q, v}, \quad\|\phi\|_{\infty}^{2} \leq 2 N_{q, v} .
$$

Proof Multiplying both sides of (4.11) by $\bar{\phi}$ and integrating by parts over the interval $[-1,1]$, then from $\mathcal{B} \phi=0$ we have

$$
\lambda \int_{-1}^{1} w|\phi|^{2}=\int_{-1}^{1}\left|\phi^{\prime}\right|^{2}+\int_{-1}^{1} q|\phi|^{2}+2 \operatorname{Re} \int_{-1}^{1} v \bar{\phi} \phi(1)
$$

This together with $\operatorname{Im} \lambda \neq 0$ yields $\int_{-1}^{1} w|\phi|^{2}=0$ and hence

$$
\int_{-1}^{1}\left|\phi^{\prime}\right|^{2}+\int_{-1}^{1} q|\phi|^{2}+2 \operatorname{Re} \int_{-1}^{1} v \bar{\phi} \phi(1)=0
$$

It follows from Lemma 4.4 and $\phi(1)=\int_{-1}^{1} \phi^{\prime}(t) \mathrm{d} t$ that

$$
\begin{aligned}
& \int_{-1}^{1} q_{-}|\phi|^{2}+2 \int_{-1}^{1}|v||\bar{\phi}||\phi(1)| \\
& \quad \leq \int_{-1}^{1} q_{-}|\phi|^{2}+2 \sqrt{2}\left(\int_{-1}^{1}|v|\right)^{\frac{1}{2}}\left(\int_{-1}^{1}|v||\phi|^{2}\right)^{\frac{1}{2}}\left(\int_{-1}^{1}\left|\phi^{\prime}\right|^{2}\right)^{\frac{1}{2}} \\
& \quad \leq \int_{-1}^{1}\left(q_{-}|\phi|^{2}+\sqrt{2} \varepsilon\|v\|_{1}|v||\phi|^{2}\right)+\frac{\sqrt{2}}{\varepsilon} \int_{-1}^{1}\left|\phi^{\prime}\right|^{2} \\
& \leq\left(\frac{1}{2}+\frac{\left\|q_{-}\right\|_{1}+\sqrt{2} \varepsilon\|v\|_{1}^{2}}{\delta}\right) \int_{-1}^{1}|\phi|^{2}+\delta \int_{-1}^{1}\left|\phi^{\prime}\right|^{2}+\frac{\sqrt{2}}{\varepsilon} \int_{-1}^{1}\left|\phi^{\prime}\right|^{2} .
\end{aligned}
$$

Setting $\varepsilon=4 \sqrt{2}$ and $\delta=1 / 4$,

$$
\int_{-1}^{1} q_{-}|\phi|^{2}+2 \int_{-1}^{1}|\nu||\bar{\phi}||\phi(1)| \leq \frac{1}{2}+4\left(\left\|q_{-}\right\|_{1}+8\|v\|_{1}^{2}\right)+\frac{1}{2} \int_{-1}^{1}\left|\phi^{\prime}\right|^{2} .
$$


Therefore, (4.12), (4.13) and $q=q_{+}-q_{-}, q_{ \pm}=\max \{0, \pm q\}$ lead us to

$$
\begin{aligned}
\int_{-1}^{1}\left|\phi^{\prime}\right|^{2} & =\int_{-1}^{1} q_{-}|\phi|^{2}-\int_{-1}^{1} q_{+}|\phi|^{2}-2 \operatorname{Re} \int_{-1}^{1} v \bar{\phi} \phi(1) \\
& \leq \int_{-1}^{1} q_{-}|\phi|^{2}+\left.2 \int_{-1}^{1}|v \|| \bar{\phi}|| \phi(1)\left|\leq \frac{1}{2}+4\left(\left\|q_{-}\right\|_{1}+8\|v\|_{1}^{2}\right)+\frac{1}{2} \int_{-1}^{1}\right| \phi^{\prime}\right|^{2}
\end{aligned}
$$

and hence

$$
\int_{-1}^{1}\left|\phi^{\prime}\right|^{2} \leq N_{q, v}
$$

where $N_{q, v}$ is defined in (4.4). Note that $\phi(x)=\int_{-1}^{x} \phi^{\prime}(t) \mathrm{d} t$ by $\phi(-1)=0$, from the CauchySchwarz inequality one sees that

$$
|\phi(x)|^{2}=\left|\int_{-1}^{x} \phi^{\prime}(t) \mathrm{d} t\right|^{2} \leq 2 \int_{-1}^{1}\left|\phi^{\prime}(t)\right|^{2} \mathrm{~d} t
$$

which together with (4.14) implies that

$$
\|\phi\|_{\infty}^{2} \leq 2 \int_{-1}^{1}\left|\phi^{\prime}(t)\right|^{2} \mathrm{~d} t \leq 2 N_{q, v}
$$

The proof of Lemma 4.5 is complete.

With the aid of the above results we prove the main results of this section.

Proof of Theorem 4.1 Multiplying both sides of (4.11) by $\bar{\phi}$ and integrating on $[x, 1]$, we have

$$
\lambda \int_{x}^{1} w|\phi|^{2}=\phi^{\prime} \bar{\phi}+\int_{-1}^{1} v \phi \overline{\phi(1)}+\int_{x}^{1}\left(\left|\phi^{\prime}\right|^{2}+q|\phi|^{2}+\nu \bar{\phi} \phi(1)\right) .
$$

Separating the imaginary parts yields

$$
\operatorname{Im} \lambda \int_{x}^{1} w|\phi|^{2}=\operatorname{Im}\left(\phi^{\prime} \bar{\phi}\right)+\operatorname{Im} \int_{-1}^{1} v \phi \overline{\phi(1)}+\operatorname{Im} \int_{x}^{1} v \bar{\phi} \phi(1)
$$

It follows from $\int_{-1}^{1} w|\phi|^{2}=0,(4.5)$ and Lemma 4.5 that

$$
\begin{aligned}
\int_{-1}^{1} \int_{x}^{1} w(t)|\phi(t)|^{2} \mathrm{~d} t \mathrm{~d} x & =\int_{-1}^{1} x w(x)|\phi(x)|^{2} \mathrm{~d} x \\
& \geq \varepsilon\left(\int_{-1}^{1}|\phi(x)|^{2} \mathrm{~d} x-\int_{\Omega(\varepsilon)}|\phi(x)|^{2} \mathrm{~d} x\right) \\
& \geq \varepsilon\left[1-m(\varepsilon)\|\phi\|_{\infty}^{2}\right] \geq \frac{\varepsilon}{2} .
\end{aligned}
$$


Then integrating (4.16) and using (4.17), Lemma 4.5, the Schwarz inequality, we have

$$
\begin{aligned}
\frac{\varepsilon}{2}|\operatorname{Im} \lambda| \leq & |\operatorname{Im} \lambda| \int_{-1}^{1} \int_{x}^{1} w(t)|\phi(t)|^{2} \mathrm{~d} t \mathrm{~d} x \\
\leq & \left|\int_{-1}^{1} \operatorname{Im}\left(\phi^{\prime}(x) \overline{\phi(x)}+\int_{-1}^{1} v(t) \phi(t) \overline{\phi(1)} \mathrm{d} t+\int_{x}^{1} v(t) \overline{\phi(t)} \phi(1) \mathrm{d} t\right) \mathrm{d} x\right| \\
\leq & \int_{-1}^{1}\left|\phi^{\prime}(x)\right||\overline{\phi(x)}| \mathrm{d} x+2 \int_{-1}^{1}|v(x)||\phi(x)||\overline{\phi(1)}| \mathrm{d} x \\
& +2 \int_{-1}^{1}|v(x)||\overline{\phi(x)}||\phi(1)| \mathrm{d} x \\
\leq & \sqrt{N_{q, v}}+4\|\phi\|_{\infty}\|v\|_{1} \leq \sqrt{N_{q, v}}+8 N_{q, v}\|v\|_{1} .
\end{aligned}
$$

Set $q_{+}(x)=\max \{0, q(x)\}$, then $|q|=q+2 q_{-}$, these facts with (4.12) lead us to

$$
\begin{aligned}
\mid \int_{-1}^{1} \int_{x}^{1}\left(\left|\phi^{\prime}(t)\right|^{2}+q(t)|\phi(t)|^{2}\right) \mathrm{d} t \mathrm{~d} x+\int_{-1}^{1}\left(\int_{-1}^{1} v(t) \phi(t) \overline{\phi(1)} \mathrm{d} t\right. \\
\left.\quad+\int_{x}^{1} v(t) \overline{\phi(t)} \phi(1) \mathrm{d} t\right) \mathrm{d} x \mid \\
=\mid \int_{-1}^{1}(x+1)\left(\left|\phi^{\prime}(x)\right|^{2}+q(x)|\phi(x)|^{2}\right) \mathrm{d} x+2 \int_{-1}^{1} v(x) \phi(x) \overline{\phi(1)} \mathrm{d} x \\
\quad+\int_{-1}^{1}(x+1)(v(x) \overline{\phi(x)} \phi(1)) \mathrm{d} x \mid \\
\leq \int_{-1}^{1}\left|\phi^{\prime}(x)\right|^{2} \mathrm{~d} x+\int_{-1}^{1}(q(x)+2 q-(x))|\phi(x)|^{2} \mathrm{~d} x+\int_{-1}^{1} 2|v(x)||\overline{\phi(x)} \| \phi(1)| \mathrm{d} x \\
=\int_{-1}^{1} 2 q_{-}(x)|\phi(x)|^{2} \mathrm{~d} x-\int_{-1}^{1} 2 \operatorname{Re}(v(x) \overline{\phi(x)} \phi(1)) \mathrm{d} x+\int_{-1}^{1} 2|v(x)\|\overline{\phi(x)}\| \phi(1)| \mathrm{d} x \\
\leq \int_{-1}^{1} 2 q_{-}(x)|\phi(x)|^{2} \mathrm{~d} x+4 \int_{-1}^{1}|v(x)||\overline{\phi(x)}||\phi(1)| \mathrm{d} x \leq 2\left\|q_{-}\right\|_{1}\|\phi\|_{\infty}^{2}+4\|v\|_{1}\|\phi\|_{\infty}^{2}
\end{aligned}
$$

which, together with the integration of (4.15), Lemma 4.5 and the Cauchy-Schwarz inequality implies that

$$
\begin{aligned}
\frac{\varepsilon}{2}|\lambda| \leq & |\lambda| \int_{-1}^{1} \int_{x}^{1} w(t)|\phi(t)|^{2} \mathrm{~d} t \mathrm{~d} x \\
\leq & \mid \int_{-1}^{1} \phi^{\prime}(x) \overline{\phi(x)} \mathrm{d} x+\int_{-1}^{1} \int_{-1}^{1} v(t) \phi(t) \overline{\phi(1)} \mathrm{d} t \mathrm{~d} x \\
& +\int_{-1}^{1} \int_{x}^{1}\left(\left|\phi^{\prime}(t)\right|^{2}+q(t)|\phi(t)|^{2}+v(t) \overline{\phi(t)} \phi(1)\right) \mathrm{d} t \mathrm{~d} x \mid \\
\leq & \left\|\phi^{\prime}\right\|_{2}+2\left\|q_{-}\right\|_{1}\|\phi\|_{\infty}^{2}+4\|v\|_{1}\|\phi\|_{\infty}^{2} \\
\leq & \sqrt{N_{q, v}}+4\left\|q_{-}\right\|_{1} N_{q, v}+8\|v\|_{1} N_{q, v} .
\end{aligned}
$$

So the inequalities in (4.7) can be obtained through (4.19) and (4.18) immediately. 
Proof of Theorem 4.2 Multiplying both sides of (4.11) by $w \bar{\phi}$ and integrating by parts on $[-1,1]$, we have from $\mathcal{B} \phi=0$

$$
\begin{aligned}
\lambda \int_{-1}^{1} w^{2}|\phi|^{2}= & \int_{-1}^{1}\left(w\left|\phi^{\prime}\right|^{2}+w q|\phi|^{2}\right)+\int_{-1}^{1} w^{\prime} \phi^{\prime} \bar{\phi} \\
& +\int_{-1}^{1}(w v \bar{\phi} \phi(1)+w(1) v \phi \overline{\phi(1)}) .
\end{aligned}
$$

Separating the imaginary parts of (4.20) implies

$$
\operatorname{Im} \lambda \int_{-1}^{1} w^{2}|\phi|^{2}=\int_{-1}^{1} \operatorname{Im}\left(w^{\prime} \phi^{\prime} \bar{\phi}+w v \bar{\phi} \phi(1)+w(1) v \phi \overline{\phi(1)}\right) .
$$

It follows from $w^{\prime} \in L^{2}[-1,1], \Lambda=\left(\int_{-1}^{1}\left|w^{\prime}\right|^{2}\right)^{1 / 2}$, Lemma 4.5 and the Cauchy-Schwarz inequality that

$$
\left|\int_{-1}^{1} w^{\prime} \phi^{\prime} \bar{\phi}\right| \leq\|\phi\|_{\infty}\left(\int_{-1}^{1}\left|w^{\prime}\right|^{2}\right)^{1 / 2}\left(\int_{-1}^{1}\left|\phi^{\prime}\right|^{2}\right)^{1 / 2} \leq \Lambda \sqrt{N_{q, v}}
$$

Using $q, v \in L^{1}[-1,1]$ and Lemma 4.5 , one sees that

$$
\begin{aligned}
&\left|\int_{-1}^{1}(w v \bar{\phi} \phi(1)+w(1) v \phi \overline{\phi(1)})\right| \leq 2\|w\|_{\infty}\|\phi\|_{\infty}^{2} \int_{-1}^{1}|v| \leq 4\|w\|_{\infty} N_{q, v}\|v\|_{1} \\
&\left|\int_{-1}^{1}\left(w\left|\phi^{\prime}\right|^{2}+w q|\phi|^{2}\right)\right| \leq\|w\|_{\infty}\left(\int_{-1}^{1}\left|\phi^{\prime}\right|^{2}+\|\phi\|_{\infty}^{2} \int_{-1}^{1}|q|\right) \\
& \leq\|w\|_{\infty} N_{q, v}\left(1+2\|q\|_{1}\right) .
\end{aligned}
$$

Recall the definition of $\eta$ in (4.6),

$$
\int_{-1}^{1} w^{2}|\phi|^{2} \geq \eta\left(\int_{-1}^{1}|\phi|^{2}-\int_{\widetilde{\Omega}(\eta)}|\phi|^{2}\right) \geq \eta\left(1-m(\eta)\|\phi\|_{\infty}^{2}\right) \geq \frac{\eta}{2}
$$

which together with (4.21), (4.22) and (4.23) yields

$$
|\operatorname{Im} \lambda| \frac{\eta}{2} \leq|\operatorname{Im} \lambda| \int_{-1}^{1} w^{2}|\phi|^{2} \leq\left(4\|w\|_{\infty}\|v\|_{1} N_{q, v}+\Lambda \sqrt{N_{q, v}}\right) .
$$

The facts (4.20), (4.22), (4.23), (4.24) and (4.25) show

$$
|\lambda| \frac{\eta}{2} \leq|\lambda| \int_{-1}^{1} w^{2}|\phi|^{2} \leq\left(\|w\|_{\infty} N_{q, v}\left(1+2\left\|q_{-}\right\|_{1}+4\|v\|_{1}\right)+\Lambda \sqrt{N_{q, v}}\right) .
$$

As a result, (4.27) and (4.26) yields the inequalities in (4.8).

\section{Conclusion}

Nonlocal boundary value problems have attracted lots of attention for the wide applications in various fields. In this paper, non-real eigenvalues of regular nonlocal indefinite Sturm-Liouville problems are considered. The existence of non-real eigenvalues of an 
indefinite Sturm-Liouville differential equation is studied. Furthermore, a priori upper bounds of non-real eigenvalues for a class of indefinite differential equation involving nonlocal point interference potential function is obtained. These results are of both theoretical and practical significance.

\section{Acknowledgements}

The authors thank the referees for his/her comments and detailed suggestions. These have significantly improved the presentation of this paper.

\section{Funding}

This research was partially supported by National Key R\&D Program of China (Grant 2017YFE0104400) and the NSF of China (Grants 11771253).

\section{Availability of data and materials}

Not applicable.

\section{Competing interests}

The authors declare that there are no competing interests.

\section{Authors' contributions}

All authors contributed equally to the writing of this paper. The authors read and approved the final manuscript.

\section{Author details}

'Department of Mathematics, Shandong University, Weihai, P.R. China. ${ }^{2}$ School of Mathematical Sciences, Qufu Normal University, Qufu, P.R. China.

\section{Publisher's Note}

Springer Nature remains neutral with regard to jurisdictional claims in published maps and institutional affiliations.

Received: 7 August 2019 Accepted: 18 October 2019 Published online: 11 November 2019

References

1. Albeverio, S., Hryniv, R.O., Nizhnik, L.P.: Inverse spectral problem for nonlocal Sturm-Liouville operators. Inverse Probl. 23, 523-535 (2007)

2. Albeverio, S., Nizhnik, L.: Schrödinger operators with nonlocal point interactions. J. Math. Anal. Appl. 332, 884-895 (2007)

3. Atkinson, F.V., Jabon, D.: Indefinite Sturm-Liouville problems. In: Kaper, H.G., Kwong, M.K., Zettle, A. (eds.) Proceedings of the Focused Research Program on Spectral Theory and Boundary Value Problems, Vol. I, pp. 31-45. Argonne National Lab. (1988)

4. Behrndt, J., Chen, S., Philipp, F., Qi, J.: Estimates on the non-real eigenvalues of regular indefinite Sturm-Liouville problems. Proc. R. Soc. Edinb. A 144, 1113-1126 (2014)

5. Behrndt, J., Katatbeh, Q., Trunk, C.: Non-real eigenvalues of singular indefinite Sturm-Liouville operators. Proc. Am. Math. Soc. 137, 3797-3806 (2009)

6. Behrndt, J., Philipp, F., Trunk, C.: Bounds on the non-real spectrum of differential operators with indefinite weights. Math. Ann. 357, 185-213 (2013)

7. Behrndt, J., Schmitz, P., Trunk, C.: Bounds on the non-real spectrum of a singular indefinite Sturm-Liouville operator on $\mathbb{R}$. Proc. Appl. Math. Mech. 16, 881-882 (2016)

8. Behrndt, J., Schmitz, P., Trunk, C.: Spectral bounds for singular indefinite Sturm-Liouville operators with $L^{1}$-potentials. Proc. Am. Math. Soc. 146, 3935-3942 (2018)

9. Behrndt, J., Schmitz, P., Trunk, C.: Spectral bounds for indefinite singular Sturm-Liouville operators with uniformly locally integrable potentials. J. Differ. Equ. 267, 468-493 (2019)

10. Catchpole, E.A.: A Cauchy problem for an ordinary integro-differential equation. Proc. R. Soc. Edinb. A 72, 39-55 (1974)

11. Curgus, B., Langer, H.: A Krein space approach to symmetric ordinary differential operators with an indefinite weight functions. J. Differ. Equ. 79, 31-61 (1989)

12. Freitas, P.: A nonlocal Sturm-Liouville eigenvalue problem. Proc. R. Soc. Edinb. A 124, 169-188 (1994)

13. Haupt, O.: Über eine Methode zum Beweis von Oszillationstheoremen. Math. Ann. 76, 67-104 (1915)

14. Kikonko, M., Mingarelli, A.B.: Bounds on real and imaginary parts of non-real eigenvalues of a non-definite Sturm-Liouville problem. J. Differ. Equ. 261, 6221-6232 (2016)

15. Krall, A.M.: The development of general differential and general differential-boundary systems. Rocky Mt. J. Math. 5, 493-542 (1975)

16. Mingarelli, A.B.: Indefinite Sturm-Liouville problems. Lect. Notes Math. 964, 519-528 (1982)

17. Mingarelli, A.B.: Volterra-Stieltjes Integral Equations and Generalised Ordinary Differential Expressions. Lecture Notes in Mathematics, vol. 989. Springer, Berlin (1983)

18. Mingarelli, A.B.: A survey of the regular weighted Sturm-Liouville problem-The non-definite case, arXiv:1106.6013v1 [math.CA]. Jun 29, 2011

19. Nizhnik, L.P.: Inverse eigenvalue problems for nonlocal Sturm-Liouville operators. Methods Funct. Anal. Topol. 15, 41-47 (2009) 
20. Nizhnik, L.P.: Inverse nonlocal Sturm-Liouville problem. Inverse Probl. 26, 125006 (2010)

21. Qi, J., Chen, S.: A priori bounds and existence of non-real eigenvalues of indefinite Sturm-Liouville problems. J. Spectr. Theory 255(8), 2291-2301 (2013)

22. Qi, J., Chen, S.: Nonlocal Sturm-Liouville eigenvalue problems and corresponding extremal problems. (submitted)

23. Reed, M., Simon, B.: Methods of Modern Mathematical Physics, IV: Analysis of Operators. Elsevier, Singapore (1972)

24. Richardson, R.G.D.: Theorems of oscillation for two linear differential equations of second order with two parameters. Trans. Am. Math. Soc. 13, 22-34 (1912)

25. Sun, F., Qi, J.: A priori bounds and existence of non-real eigenvalues for singular indefinite Sturm-Liouville problems with limit-circle type endpoints. Proc. R. Soc. Edinb. A 1-13 (2019)

26. Sun, F., Qi, J.: The spectral problems of nonlocal indefinite Sturm-Liouville operators. (submitted)

27. Wentzell, A.D.: On boundary conditions for multidimensional diffusion processes. Theory Probab. Appl. 4, 164-177 (1959)

28. Xie, B., Qi, J.: Non-real eigenvalues of indefinite Sturm-Liouville problems. J. Differ. Equ. 8(8), 2291-2301 (2013)

29. Zettl, A.: Sturm-Liouville Theory. Math. Surveys Monogr., vol. 121. Am. Math. Soc., Providence (2005)

\section{Submit your manuscript to a SpringerOpen ${ }^{\circ}$ journal and benefit from:}

- Convenient online submission

- Rigorous peer review

Open access: articles freely available online

High visibility within the field

Retaining the copyright to your article

Submit your next manuscript at $\gg$ springeropen.com 